\title{
Whole-Body-MR-Diffusion Weighted Imaging in Oncology
}

\author{
Ganzkörper-MR-Diffusionsbildgebung in der Onkologie
}

Authors

Affiliation
T. Wilhelm, B. Stieltjes, H. P. Schlemmer

Radiology, German Cancer Research Center, Heidelberg
Key words

- MR-diffusion/perfusion

- metastases

treatment effects

eingereicht 12.9 .2012

akzeptiert 18.3.2013

Bibliography

DOI http://dx.doi.org/

10.1055/s-0033-1335428

Published online: 2.9.2013

Fortschr Röntgenstr 2013; 185:

950-958 @ Georg Thieme

Verlag KG Stuttgart · New York .

ISSN 1438-9029

\footnotetext{
Correspondence Dr. Thomas Wilhelm

Radiologie, Deutsches Krebsforschungszentrum Im Neuenheimer Feld 280 69120 Heidelberg Tel.: ++ 49/6221/42 2491

Fax: ++ 49/62 21/42 2492

thomas.wilhelm@dkfz-

heidelberg.de
}

\section{Abstract \\ $\nabla$}

The clinical implementation of whole body diffusion weighted imaging (WB-DWI) for tumor-detection, -characterization and therapy monitoring is well underway. The method is fast, robust and combined with its wide availability on modern MRI scanners, it has a vast potential clinical impact. Owing to the high tumor to background contrast, its main application areas are simple detection of tumor suspicious lesions (primary tumor, recurrence, and metastasis), tumor grading and therapy monitoring. WB-DWI has a strong diagnostic potential regarding the evaluation of bone marrow and its diseases and as thus, tumor detection and therapy monitoring of bone metastasis is of particular interest. The assessment of a lymphatic tumor spreading is constricted. One of the major hurdles that still hamper the wide clinical application of WB-DWI is a lack of standardization of measurement parameters that limit the comparability of current research results.

Key Points:

- Owing to the high tumor to background contrast WB-DWI allows fast assessment of tumor distribution and total tumor burden.

- WB-DWI allows therapy monitoring.

- WB-DWI is widely available.

Citation Format:

- Wilhelm T, Stieltjes B, Schlemmer HP. WholeBody-MR-Diffusion Weighted Imaging in Oncology. Fortschr Röntgenstr 2013; 185: 950-958

\section{Zusammenfassung}

$\nabla$

Die Ganzkörper-Diffusionsbildgebung (GK-DWI) befindet sich aktuell auf dem Weg, ein praxistau- gliches Instrument der Detektion und Charakterisierung von Tumoren und des Therapiemonitorings zu werden. In Zukunft ist aufgrund der robusten Technologie, der schnellen Durchführbarkeit und der Möglichkeit der Implementierung der entsprechenden Protokolle an den meisten modernen MRT-Geräten eine weitverbreitete klinische Anwendung zu erwarten. Die Anwendungsmöglichkeiten liegen aufgrund des hohen Tumorzu-Normalgewebe-Kontrasts in der vereinfachten Detektion tumorsuspekter Läsionen (Primärtumor, Rezidiv, Metastasen) sowie im Tumorgrading und im Therapiemonitoring. Besondere Eigenschaften sind der GK-DWI in der diagnostischen Beurteilung sowie im Therapieansprechen des Knochenmarks und seiner Erkrankungen zuzuschreiben, sodass ein potenziell großes Anwendungsgebiet in der Detektion und dem Therapiemonitoring ossärer Tumorausbreitungen liegen könnte. Die Beurteilung einer lymphatischen Tumorausbreitung ist nur eingeschränkt möglich. Das Hauptproblem in der praktischen Umsetzung der GK-DWI besteht aktuell allerdings noch in der fehlenden Standardisierung der Sequenzparameter und der damit verbundenen fehlenden Vergleichbarkeit von Studienergebnissen.

\section{Introduction \\ $\nabla$}

For about the last 15 years, high-resolution whole body MRI has yielded relevant information on local and systemic growth in oncological diagnostic testing when employed over the course of examination [1-3]. In this context, diffusion imaging initially played no significant role.

In the late $1990 \mathrm{~s}$ it established itself as a neuroradiology-based tool for diagnosing ischemia [4].

The development of rapid echoplanar sequences (EPI), more powerful and faster gradient 
systems, parallel acquisition techniques (PAT) with multichannel coils and receiver technology facilitated a more robust image acquisition in the trunk [5]. In 2004 Takahara et al. made a pioneering contribution to the development of whole-body diffusion-weighted imaging, by introducing a technology called DWIBS (diffusion-weighted whole-body imaging with background suppression), the acquisition of which can be performed during free respiration, that suppresses background signals and allows examination of the whole body within 25 minutes [6]. In the meantime, this technique has become viable for broad clinical application, since the appropriate protocols can be implemented on the majority of MRI systems [7]. Whole-body DWI supplements the conventional whole-body MRI sequence protocol for various tumor entities, where the accuracy depends on the particular tumor entity in some cases:

1. diffusion-weighted images with high b-values have a high tumor-to-normal tissue contrast, thereby improving the detectability of tumor lesions as well as the infiltrative spread thereof.

2. Diffusion-weighted images and parameter cards of the diffusion constants provide clues about tissue architecture such as e.g. cell density and cellular membrane integrity and correlate with grading [8]. More in-depth information on the proliferation activity and micromilieu for the purpose of individual tumor characterization can be gathered from additional measurements of tumor blood supply by means of dynamic contrast-enhanced MRI (DCE) and - particularly in the case of brain tumors and prostate carcinoma - metabolism by means of MR spectroscopy.

3. DWI can be consulted as predictive and quantitative biomarker for individualized oncological therapy and constitutes an important diagnostic tool for testing new active substances (targeted drugs) [9]. ADC provides a quantitative progression parameter for monitoring therapy. Additionally, when frequent follow-up examinations are performed, it is advantageous to forego the use of ionizing rays and intravenous contrast agents, which has no effect particularly when examining young patients who require frequent follow-up and on kidney failure patients in the course of frequent therapy monitoring or for early recurrence testing of potentially tumor-free patients [10].

4. For better overview, $3 \mathrm{D}$ images can be generated [11] that provide similar imaging results as nuclear medicine procedures. In this way, DWI also supports the feasibility of full body MRI, since economics dictate that the time and human resources required for examination, evaluation and reporting must be kept in check without, however, compromising the quality of the examination results.

This survey article explains the mechanisms of the appearance of contrast and focuses on the current clinical uses of whole-body DWI for the systematic diagnosing of tumor spread, without addressing in detail the individual organ systems already examined in numerous original articles.

\section{Measuring diffusion with NMR}

$\nabla$

Owing to their thermal energy, water molecules are in constant motion (Brown's molecular motion). The scope of movement in the body is tissue-specific and is ascertained by means of free length of travel or the diffusion constant. The principle of measuring diffusion by means of diffusionweighted MRI involves first dephasing and then rephasing the transverse magnetization of excited water protons along diffusion gradients. Overall, the net magnetization of stationary water protons experiences no change, while that of protons in motion is decreased due to residual dephasing. The strength of signal loss in this process is function of the free length of travel of the water molecules in the particular tissue.

Precise mathematical computation of the complex diffusion processes in tissue and the measurement thereof using MR is possible only within limits and does not allow a simple relationship to be derived between, for example, cell density and diffusion constant. Ideal free diffusion in liquids can be mathematically described using the free diffusion constant $D_{\mathbf{O}}$. In the case of free diffusion, the signal decreases according to $S=S_{\mathrm{O}} \exp \left(-b D_{\mathrm{O}}\right)$, where $S$ represents the signal with diffusion gradients and $S_{\mathbf{O}}$ the starting signal. The $b$ value is provided by the gradient amplitude $G$ and the temporal gradient profile (expressed by $\Delta$ and $\delta$ ). The free parameter $D_{\mathrm{O}}$ can be computed from $S$ and $S_{\mathrm{O}}$ by assuming a monoexponential drop in signal. If, however, there are restrictions caused by cell walls, then the signal decreases as $\mathrm{S}=S_{\mathrm{O}} \exp \left(-b D_{a p p}(t)\right)$ if $b$-values are low (roughly $b \leq 1000 \mathrm{~s} /$ $\mathrm{mm}^{2}$ ). In this case, $D_{a p p}$ is what is known as the "Apparent Diffusion Coefficient" (ADC), the value of which is roughly $1 \mu \mathrm{m}^{2} / \mathrm{ms}$ in human tissue. It should be kept in mind, however, that $D_{a p p}(t)$ is different from $D(t)$, since the diffusion gradients are ultimately long. It should also be kept in mind that $D_{\text {app }}(t)$ is a function of the temporal course of the gradient profile. For example, the same $b$-value can be generated with, for example, a short $\Delta$ or a very long $\Delta$, if $\delta$ is changed. $D_{\text {app }}(t)$ is generally independent of these parameters, however. Despite this fact, $D_{\text {app }}(t)$ or ADC can usually be regarded as a good approximation for $D(t)[12]$.

\section{Acquisition, contrast mechanisms and image interpretation}

$\nabla$

\section{Acquisition}

Whole-body DWI can be performed on any MRI system with a field strength of at least $1.5 \mathrm{~T}$. While diffusion imaging has proven to be more reliable at $1.5 \mathrm{~T}$ than $3 \mathrm{~T}$ owing to the more homogenous fat suppression in a wider field of view (FOV) and the lower occurrence of susceptibility artifacts, there is potential for improvement in the signal-tonoise-ratio (SNR) at $3 \mathrm{~T}$. Using ultra-fast echo-planar-imaging (EPI) sequences with an echo time $<80 \mathrm{~ms}$ facilitates a favorable signal-to-noise ratio. Either integrated body coils or whole body surface coils can be used for signal reception. Using whole body surface coils can allow a scan area of approximately $200 \mathrm{~cm}$ at higher image quality without requiring the repositioning of the patient or coil system during the examination. Examination is performed during free respiration with as many table positions as necessary to image the body from the top of the head to mid-thigh. 0 Table 1 provides an overview of the sequence parameters for singleshot-spin-echo echo planar MR imaging (EPI) performed during free-respiration [13]. The acquired diffusion-weighted images with high b-values are usually reconstructed in 


\begin{tabular}{|l|l|}
\hline parameter & value \\
\hline repetition time(ms) & 9000 \\
\hline echo time $(\mathrm{ms})$ & 68 \\
\hline number of slices & 200 ; four stations, 50 slices per station \\
\hline field of View (FoV) & $45 \times 45 \mathrm{~cm}$ (variable) \\
\hline matrix & $128 \times 128$ interpolated to $256 \times 256$ \\
\hline number of acquired signals & six \\
\hline fat suppression & short-tau inversion recovery (inversion time 180 ms) \\
\hline diffusion coding & trapezoidal gradients, trace-weighted, bipolar gradients \\
\hline parallel imaging & IPAT factor 2 ; number of reference lines: 30 \\
\hline slice thickness $/$ gap $(\mathrm{mm})$ & $5 / 0$ \\
\hline b-values $\left(\mathrm{sec} / \mathrm{mm}^{2}\right)$ & 50,800 \\
\hline bandwidth $(\mathrm{Hz} / \mathrm{pixel})$ & 1628 \\
\hline
\end{tabular}

Table 1 Sequence parameters for Single-Shot-Spin-Echo EchoPlanar MR Imaging (EPI) during free-respiration [14]. thin (4-5 mm) multiplanar slices or as Maximum Intensity Projections (MIPs). Advanced software algorithms additionally allow diffusion-weighted images to be merged with morphological sequences.

\section{Contrast mechanisms and image interpretation}

Contrast depends on the selection of b-values during image acquisition, since the contrast intensity increases when higher b-values are selected owing to an improved contrast-to-noise-ratio (CNR). At the same time, selection of the maximum b-value is limited by the signal-to-noise ratio. In the case of oncological patients, the visual assessment of images with high b-values is crucial for detecting and, in some cases, characterizing lesions associated with tumors. What is known as the T2-shine-through-effect constitutes the problem that describes the relationship between signal intensity and transversal T2 relaxation time. When b-values are low, structures especially with long T2 time pose a potential source of errors. The simplest way of preventing misdiagnoses due to the T2-shine-through effect is acquiring multiple b-values to facilitate a quantitative evaluation. For this purpose, it is necessary to acquire at least two b-values, the corresponding signal amplitudes thereof must be adapted to the behavior of a simple mono-exponential curve [14]. This procedure allows the computation of an ADC value (apparent diffusion coefficient) that is independent of the magnetic field strength and the T2-shine-through-effect. An evaluation can be performed on the basis of either a region of interest (ROI), a volume of interest (VOI) or a pixel-for-pixel analysis [15]. There is additionally the risk of generating false-positive findings in movement-dependent anatomical regions such as the mediastinal structures including the hili, the portions of the left hepatic lobe located in close topographical relation to the heart as well the ventral thoracic chest wall and regions in immediate proximity to the air interfaces such as in the neck and shoulder region.

\section{Normal image}

$\nabla$

To be able to use whole-body diffusion imaging diagnostically for tumor detection and therapy monitoring, familiarity with the normal images of soft tissue and bone marrow is imperative. Findings yielded by DWI should be correlated with conventional sequences. As $\bullet$ Fig. 1 shows, several structures such as the CNS, the salivary glands, the spleen, the lymph node stations as well as the organs of the urinary tract and, depending on the female patient's cycle, the normal female breast parenchyma as well as hepatic cysts and hemangiomas exhibit a physiologically induced T2-shinethrough-effect as a result of a long T2-time and thus appear hyperintense in diffusion-weighted images [16]. If the water content of a lesion increases following a clearing reaction of dead cells or due to tumor necrosis it would appear as hyperintense owing to the T2-shine-through-effect in low b-image. Post-interventional changes to the tissue such as interstitial edema, surgical interventions and inflammatory reactions following irradiation likewise have a hyperintense appearance at low b-values and can thus result in false positive findings. The variability of the distribution of red and yellow bone marrow can be excellently visualized using this method. In diffusion weighted images, yellow bone marrow has a lower signal than red bone marrow due to its lower cellularity [17]. Red bone marrow, in contrast, emits a more hyperintense signal owing to its higher cell density [18]. The atrophy of red bone marrow above the age of 40, especially in women (apparently due to the estrogen deficit and osteoporosis), and obesity cause signal intensity to drop as age increases. From an oncological viewpoint the effect of cytotoxic chemotherapies plays an important role, since, owing to the decreasing cellularity of the bone marrow, they bring about a drop in signal as a result of the cytotoxic effect with subsequent bone marrow fattening. Conversely, administering granulocyte colony-stimulating factors (G-CSF) during chemotherapy in response to rising cellularity and the associated rise in signal can mimic tumor progress.

\section{Tumor detection (qualitative analysis)}

The sensitivity of DWI in detecting tumors depends on the tumor entity, tumor grading and the anatomical localization [8]. A hyperintense appearance was demonstrated in diffusion-weighted images for a number of entities such as prostate carcinoma ( $\bullet$ Fig. 2), breast carcinoma [19], gynecological tumors such as cervical and ovarian carcinoma, hepatocellular carcinoma (HCC), rectal carcinoma and other small cell tumors such as neuroendocrine tumors ( $\odot$ Fig.3), small cell tumors and many pediatric tumors such as hepato-, nephro- and neuroblastoma [20, 21]. The same signal behavior is also exhibited for hematological tumors such as multiple myeloma and lymphoma [13]. Similarly, the metastases also appear hyperintense in the diffusion-weighted images at high b-values [13]. In contrast, renal primary tumors as well as the metastases thereof are more difficult to see. Clear cell metastases in 


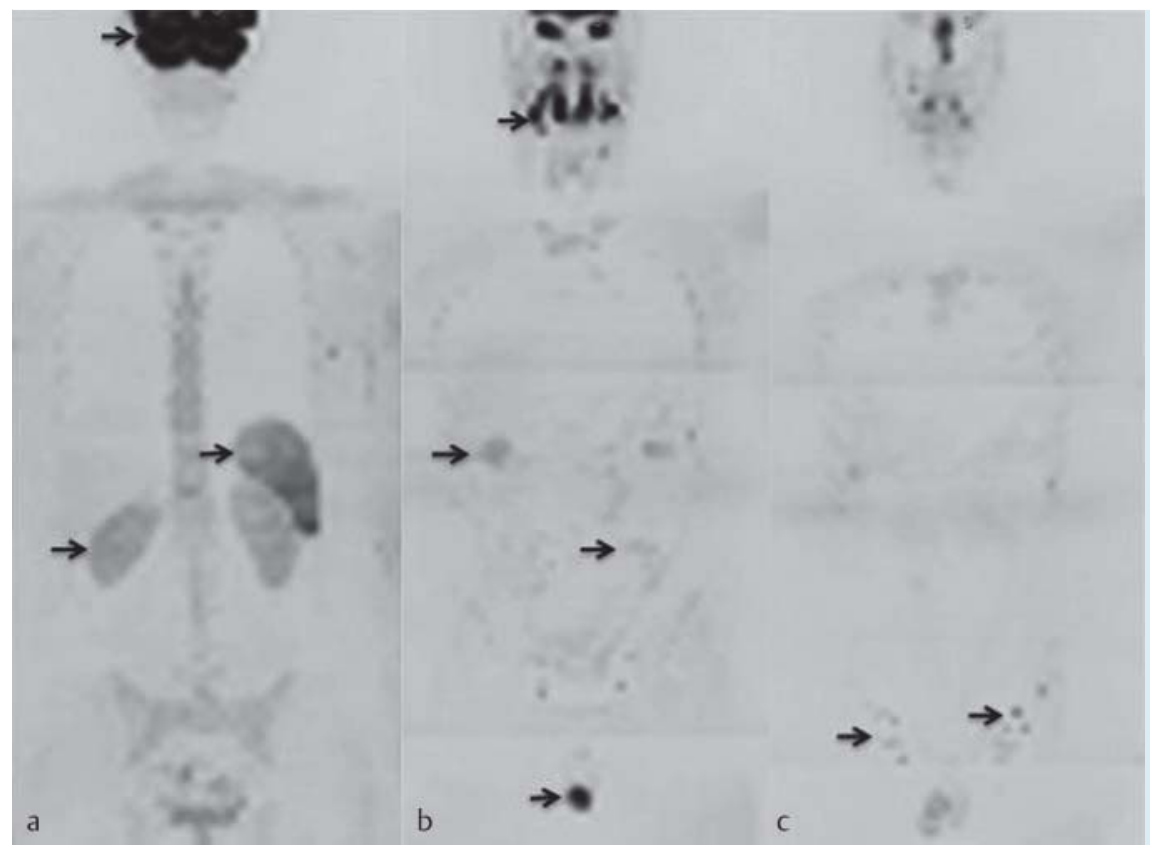

Fig. 1 Normal appearance of organs in coronary DWI, inverted in a 25-year old healthy patient. Some organs like the salvary glands, the nervous system, the lymphatic system, the urogenital system and the gastrointestinal system indluding the gallbladder show physiologic T2-shine-through effect owing to a long T2-value.

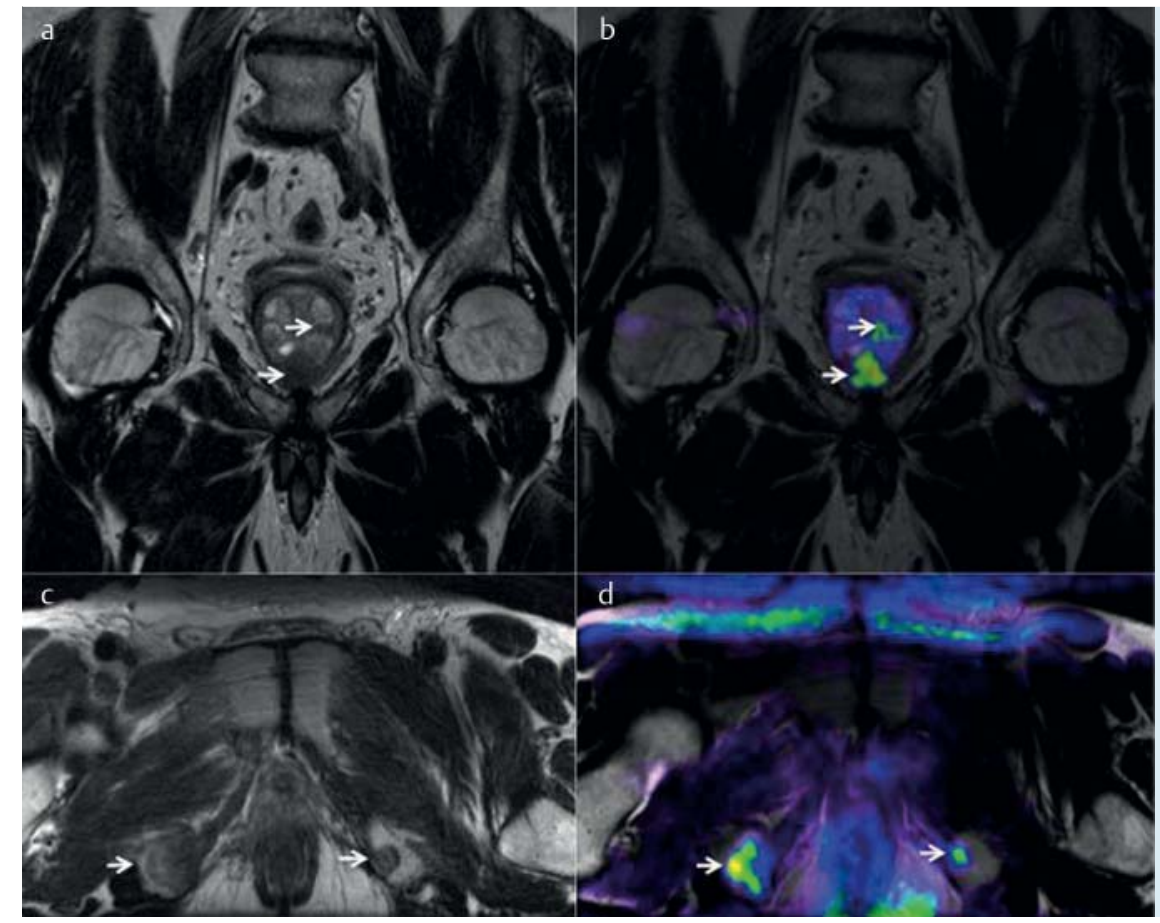

Fig. 2 68-year old patient with multifocal postate cancer and osseas metastasis. The figures $\mathbf{a}$ and $\mathbf{b}$ show T2-weighted images in coronary $\mathbf{a}$ and axial $\mathbf{b}$ orientation. The figures $\mathbf{c}$ and $\mathbf{d}$ show hybrid pictures of diffusion and T2-weighted images in coronary $\mathbf{a}$ and axial $\mathbf{b}$ orientation.

particular often come across as unremarkable in diffusion imaging compared to other histological types [22]. Tumors of primarily normal histological structure as well as well differentiated or tumors of low malignancy are likewise occasionally difficult to detect [23].

Owing to their increased cellularity relative to the surrounding bone marrow tissues, osteolytic tumors are highly visible with diffusion imaging. False-positive findings are caused primarily by degenerative changes, fractures, edemas, infections, hemangiomas and dispersed red bone marrow [13]. More precise differentiability can be achieved by using the ADC value and conventional anatomical imaging.
False-negative results in the detection of bone marrow tumors arise in the case of low-grade tumor infiltration of the bone marrow at the level of the base of the skull as well as the cranial vault as a result of the physiologically high signal intensity of the brain and in the case of metastases that develop in hypercellular bone marrow. Osteolytic bone metastases are more visible in diffusion imaging than primary sclerotic lesions or those induced by therapy, which can evade detection due to their low cell count and a low extracellular water content [20].

Assessing lymph nodes in cancer patients is generally difficult. Normally, lymph nodes are evaluated according to size, 

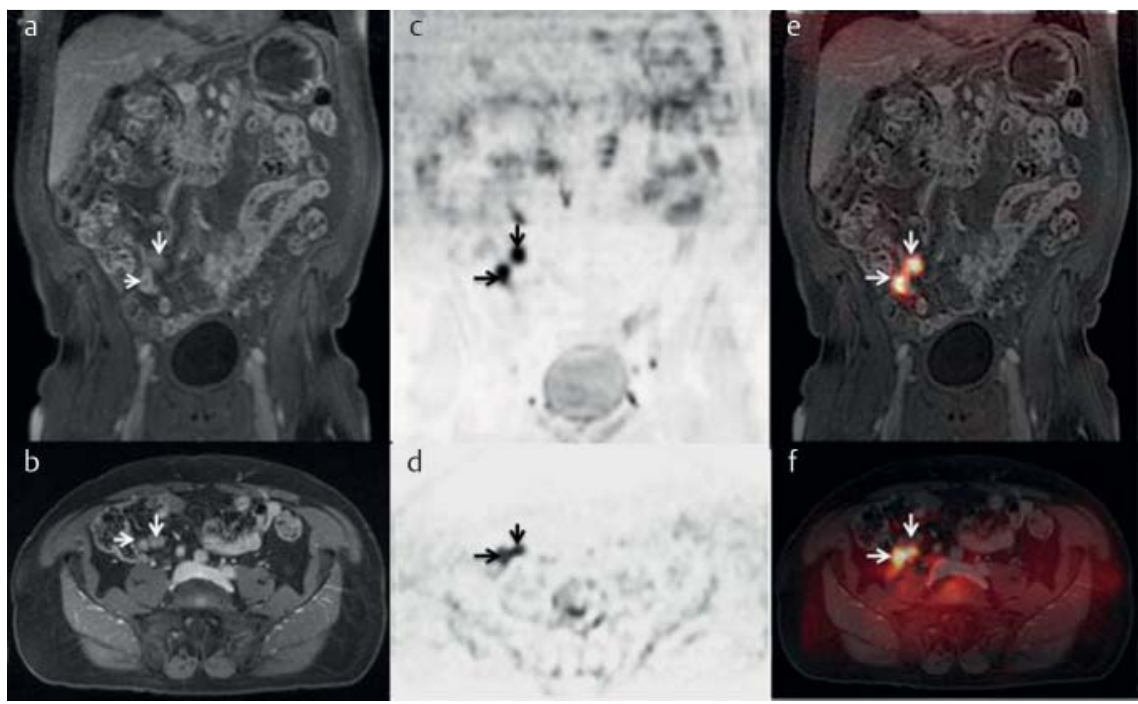

Fig. 3 a T1-w post gadolinium in coronary orientation. b T1-w post gadolinium in axial orientation. c DWI in coronary orientation, inverted. $\mathbf{d}$ DWI in acial orientation, inverted. e, $\mathbf{f}$ Coronary $\mathbf{c}$ and axial $\mathbf{f}$ hybrid image of T1-w post gadolinium and DWI in a 75-year old patient with appendic carcinoid and local lymphatic metastasis (s. $\rightarrow$ ).

which, however, provides only limited information on the tumor infiltration of lymph nodes ( $\bullet$ Fig.4). Because of the overlapping values in the threshold range, the ADC value does not allow clear differentiation between malignant and benign lymph nodes [7], even if a few studies have established that metastatic lymph nodes exhibit a significantly lower ADC than their healthy counterparts [24-27]. An additional study shows that while DWI is inferior to FDG PETCT in detecting primary colorectal carcinomas, it is superior in detecting lymph node metastases [28]. Patients with lymphoma diseases are an exception, with a study having established that nodal and extranodal manifestations of a non-Hodgkin's lymphoma can be detected with certainty using diffusion imaging and that significant changes in ADC can be observed in patients under therapy, even at early stage, before the affected lymph nodes change in size.

\section{Tumor characterization (quantitative analysis)}

Whole-body diffusion-weighted imaging is of limited suitability for characterizing soft tissue lesions if merely the signal intensity is examined for evaluation without taking into consideration the morphological appearance and the ADC ( $\bullet$ Table 2). Only when examined together with anatomical localization and ADC can the tissue be characterized so that grading can be performed if certain tumorous diseases are present. For example it was demonstrated that there is a correlation between ADC and histopathological differentiation (Gleason degree) in the case of prostate carcinoma, with moderately differentiated prostate carcinomas (Gleason 6) exhibiting higher ADC values in the peripheral zone than mildly (Gleason 7) or poorly differentiated (Gleason 8) prostate carcinomas [30, 31]. Tumor grading is also possible for additional tumor entities such as glial tumors, hepatocellular carcinoma (HCC), bladder carcinoma and rectal carcinoma [32-34].

\section{Therapy monitoring}

$\nabla$

The complex changes in tumor tissue contrast over the course of therapy are a function of the particular therapy and the passage of time. In general, tumors with rising wa- ter content, e.g., due to interstitial edema, following lysis, necrosis or inflammations, appear increasingly hyperintense on diffusion-weighted images with low b-values (T2shine-through-effect) and ADC cards (increasing diffusion). The observation of an increase in signal intensity alone on the b-images must not lead to false-positive findings. Each cytotoxic process that results in a necrosis or apoptosis on the cellular level exhibits, as early reaction owing to the failure of the sodium-potassium pump, an influx of water intracellularly and thereby a cytotoxic cellular edema that results in a drop in ADC. If, over the course of time, the cell membrane exhibits increased permeability resulting from the intracellular edema and shifting of the oncotic pressure, water flows from the intracellular space to the extracellular space [35]. This results in a drop in signal intensity in the diffusion-weighted images with high b-values and a corresponding increase in $A D C$ value $[9,36,37]$. On the other hand, tumor progression is reflected, in addition to size progression, in the appearance of new cell-dense areas with correspondingly hyperintense signal or in the form of an increase in signal intensity in the diffusion-weighted images. Cell death resulting from response to therapy brings on a change in the diffusion-weighted image prior to the change in lesion size. Diffusion imaging can therefore be considered to be useful tool for the early measurement of response to therapy [13, 38, 39]. Increases in ADC that can be associated with the death of tumor cells have been found to be inconstant over the course of time. A drop in ADC can be expected in the process of a clearing reaction of dead cells by macrophages and subsequent tissue reconstruction, e.g. through fibrosis. This is a potential source of error posed by diffusion-weighted imaging, since both the tissue reconstruction during the healing process and a tumor recurrence can bring about a drop in ADC. $\odot$ Fig. 5 provides an overview of the influence of therapy on ADC on the cellular level.

\section{Therapy-induced changes in bone marrow}

Because T1- and T2-weighted MR images often show no substantial changes with regard to the evaluation of bone marrow over the course of treatment of bone marrow diseases, these purely morphological sequences would appear to have only limited use for monitoring therapy [40]. 


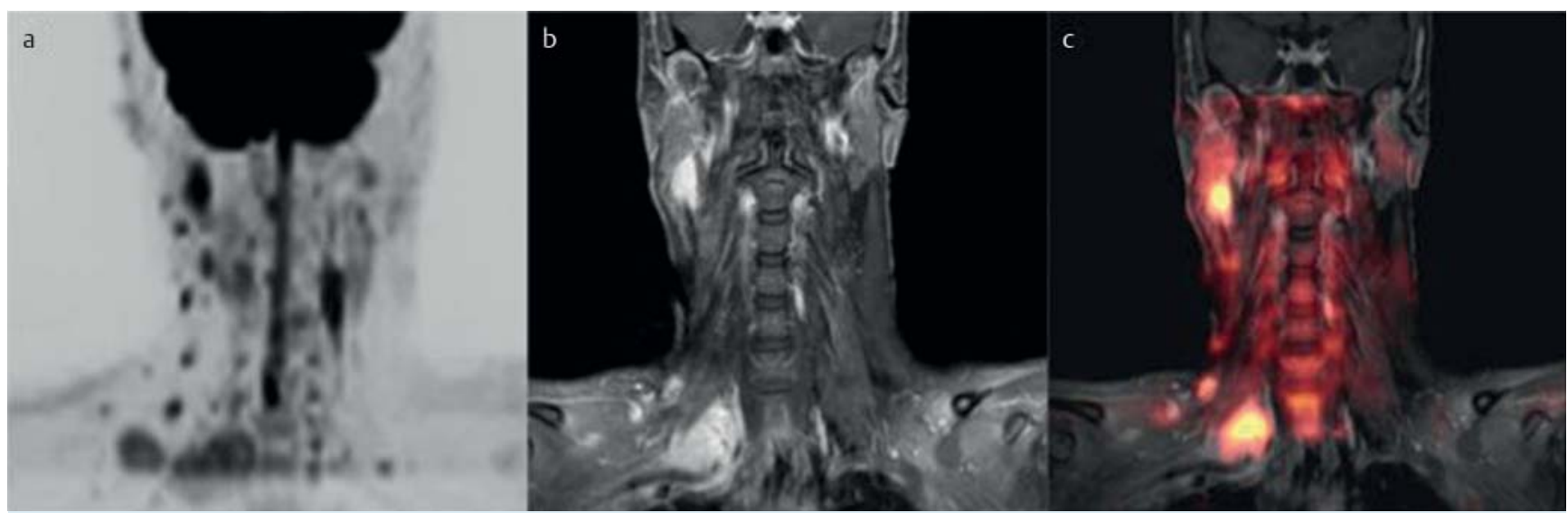

Fig. 4 a Coronary 3D-Reconstruction of an inverted DWI of the neck, $\mathbf{b}$ coronary T1-w post-gadolinium of the neck, $\mathbf{c}$ Hybrid-image of T1-w post-gadolinium and inverted coronary DWI of the neck in a patient with lymphatic metastasis in thyroid cancer.

Table 2 Signal behavior in diffusion-weighted imaging.

\begin{tabular}{lll}
$\begin{array}{l}\text { signal intensity at } \\
\text { high b-values }\end{array}$ & ADC & interpretation \\
\hline$\uparrow$ & $\downarrow$ & $\begin{array}{l}\text { hypercellular tumor, cytotoxic edema (DD oc- } \\
\text { casionally abscess, viscous fluid, hematoma) }\end{array}$ \\
\hline$\uparrow$ & $\uparrow$ & $\begin{array}{l}\text { T2-shine-through-effect pronounced at low } \\
\text { b-values }\end{array}$ \\
\hline$\downarrow$ & $\uparrow$ & $\begin{array}{l}\text { low-protein fluids, necrosis, occasionally well } \\
\text { differentiated, hypocellular adenocarcinoma }\end{array}$ \\
\hline$\downarrow$ & $\begin{array}{l}\text { susceptibility artifacts, fibrotic tissue, muscle } \\
\text { tissue, bones, adipose structures }\end{array}$ \\
\hline
\end{tabular}

\section{Diffuse infiltration of bone marrow}

This is where diffusion imaging provides an added clinical benefit, since it can show that in patients with leukemia, for example, there is a correlation between an increase in ADC and successful therapy [41]. 0 Fig. 6 shows a response to therapy using a patient with multiple myeloma as an example. In contrast, patients not experiencing clinical improvement exhibit persisting hyperintensities in diffusionweighted imaging with high b-values, which suggests an ongoing tumor-induced hypercellularity. While the longterm changes in bone marrow observed following successful treatment of the underlying disease depend on the type of tumor and treatment plan, the signal intensity and ADC slowly decline in the months following the start of therapy in most cases [42]. Like the elapsing pathophysiological process, signal alterations in diffusion imaging accompanying the curing of bone marrow disease have not yet been explained in detail. It is apparent, however, that effects such as sclerosis, clearing reactions of dead tumor cells, repopulation of (usually yellow) bone marrow, chemotherapy-induced myelofibrosis and decreased perfusion lead to a reduction in signal intensity and ADC [43].

\section{Focal infiltration of bone marrow}

Studies examining neoadjuvant response to therapy in bone tumors such as osteosarcoma likewise show significant differences in $A D C$ between patients responding to therapy, who exhibit correspondingly higher ADC values after therapy, and patients not responding to therapy [44]. However, the signal may still remain high in diffusion-weighted imaging despite response to therapy and the associated necrosis. This is due to the T2-shine-through-effect, which appears particularly in the case of bone marrow diseases such as multiple myeloma with focal or diffuse infiltration pattern and in the case of lymphomas, but also occasionally with other solid metastatically dispersing neoplasms. Nevertheless, the T2shine-through-effects are comparatively easy to detect through the ADC map, which in this case exhibits very high values when viewed together with T2-weighted sequences or short-tau-inversion-recovery (STIR).

\section{Reaction in soft tissue}

Rises in ADC following successful chemo and radiation therapy have been documented for several anatomical regions, including breast carcinoma, primary and metastatic liver tumors as well as for primary osteosarcoma and malignant brain tumors [13]. Just three to seven days after their first chemotherapy session, patients with hepatic metastasis of stomach and colorectal carcinomas exhibit a change in ADC correlating to a response to therapy [45]. Several clinical and pre-clinical studies have examined how radiation impacts ADC, showing that ADC increases rather rapidly in the case of radiation-sensitive tumors. In one pre-clinical study, proton MR spectroscopy detected visible changes in ADC after only 24-72 hours without any signs of the lesion changing size, but coinciding with a metabolic disturbance of the tumor tissue [46]. Rises in ADC appear incrementally with fractionated radiation therapy, where the greatest visible changes can be observed consecutively at the end of therapy $[47,48]$. This effect can most likely be attributed to the death of tumor cells, the development of interstitial edema resulting from radiogenic inflammatory reactions and increased vascular permeability. Overall, it can be postulated from a number of studies that the absence of a rise in $\mathrm{ADC}$ following the conclusion of the early reaction with the formation of intracellular edema under chemotherapy, radiation therapy or a combination thereof suggests a poor response to therapy. 


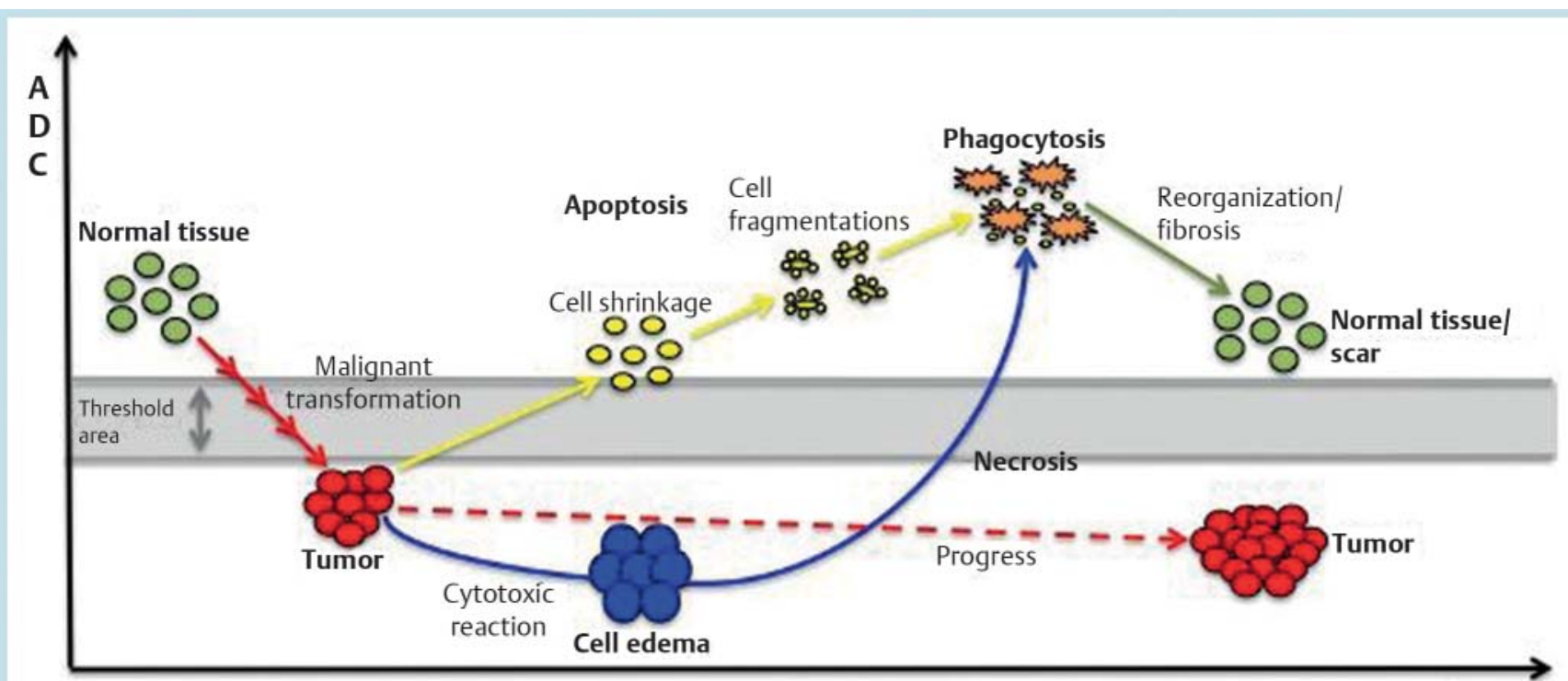

Time

Fig. 5 The influence of a therapy on the development of ADC on cellular level.

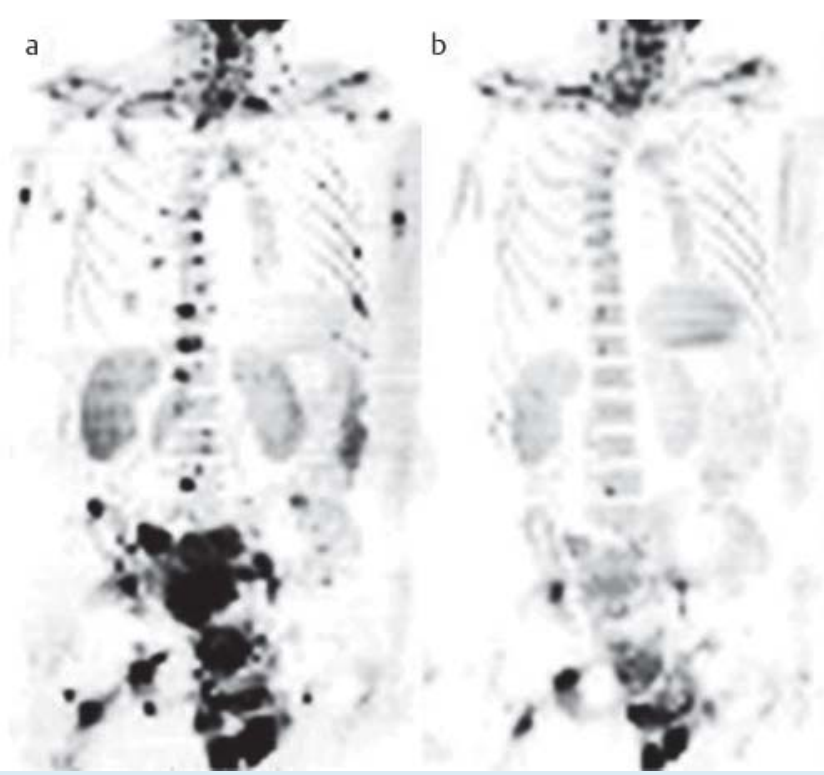

Fig. 6 62-year old patient with multiple myeloma. The diffusion-weighted whole body-MRI before $\mathbf{a}$ and 3 months after chemotherapy $\mathbf{b}$ shows therapy response in osseus lesions.

\section{Comparability of whole-body DWI and FDG-PET $\nabla$}

Fluorodeoxyglucose (FDG) PET and whole-body DWI are assuming a complementary role in oncological evaluation, since they are able to illustrate different tissue properties. While FDG PET presents metabolic information on intracellular glucose uptake, diffusion imaging primarily represents microstructural information. The FDG uptake in PET for diagnostic purposes and therapy monitoring is typical for several FDG-positive entities such as bronchial cancer, rectal cancer and lymphoma and is advantageous over DWI in evaluating structures such as the mediastinum, the lungs and the spleen [49]. However, a number of tumors such as prostate carcinoma, hepatocellular carcinoma and glial tumors may prove to be FDG-negative depending on their degree of differentiation [50-52]. Precisely in the case of these tumors with a low FDG uptake, which also include neuroendocrine tumors, thyroid gland carcinomas and several types of low-malignancy lymphoma, diffusion images with high b-values are advantageous for detection [53]. Diagnostic advantages of diffusion-weighted imaging are also apparent in anatomical regions that have a heavy accumulation of FDG in healthy tissues such as in the brain, liver, bone marrow and urinary system. Several studies show an inverse correlation between the standardized uptake value (suv) of PET and ADC $[54,55]$. Because the two procedures complement one another, combining FDG-PET and wholebody DWI through PET-MRT could constitute a further advancement in the detection and characterization of tumorassociated lesions [56] as well as in the evaluation of response to therapy [57].

\section{Indications for whole-body diffusion-weighted imaging in oncology}

Whole-body diffusion-weighted imaging makes it possible to show the primary location and remote metastasis of many types of tumors within the limits of the particular accuracy available [5]. Suitable candidates would appear to be tumors with small cell and cell-dense appearance under histological examination, such as multiple myeloma, malignant myeloma ( $\bullet$ Fig. 7), neuroendocrine tumors, small cell tumors and pediatric tumors [13]. Whole-body diffusionweighted imaging is beneficial in the case of bone marrow diseases as well as for evaluating therapy response in patients with mixed osteoplastic-osteolytic metastasis patterns, particularly breast and prostate carcinoma. The method is additionally advantageous for instances in which the use of ionizing radiation must be decreased to a minimum, such as for children and pregnant women 


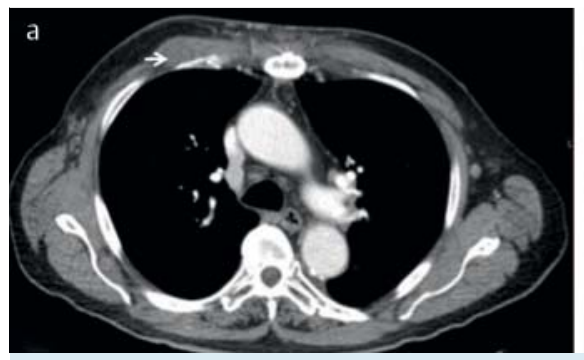

b

Fig. 7 74-year old patient with soft tissue metastasis of a malignant melanoma in the major pectoralis muscle (s. $\rightarrow$ ). a shows a computed tomographic of the thorax with contrast media. The soft tissue metastatis was not detected here. $\mathbf{b}$ shows te corresponding slice of inverted DWI. Here the lesion can be detected clearly. $\mathbf{c}$ shows a hybrid picture of computed tomography and DWI.
[58 - 60] as well as when frequent follow-up examinations are necessary. It is likewise imperative to minimize the risk of inducing radiogenic secondary tumors in tumor patients who have the prospect of a long life expectancy following a curative therapy approach. Patients who cannot undergo contrast-enhanced CT examination due to impaired kidney functions or allergies can also benefit from this diagnostic imaging technology. With its higher tumor-to-normal tissue contrast, whole-body diffusionweighted imaging holds further potential for tumor detection, grading and therapy monitoring, while being easier and more cost-effective to perform. However, validation through multicentric studies is currently limited by the lack of standardization of methods.

\section{Note}

\section{$\nabla$}

In the eFirst-version of this article the sequence of the figures $7 \mathrm{~b}$ and $7 \mathrm{c}$ was wrong. It has been corrected.

\section{References}

1 Schlemmer HP et al. Fast whole-body assessment of metastatic disease using a novel magnetic resonance imaging system: initial experiences. Invest Radiol 2005; 40: 64-71

2 Ruehm SG et al. Whole-body MRA on a rolling table platform (AngioSURF). Fortschr Röntgenstr 2000; 172: 670-674

3 Eustace $S$ et al. A comparison of whole-body turboSTIR MR imaging and planar 99mTc-methylene diphosphonate scintigraphy in the examination of patients with suspected skeletal metastases. Am J Roentgenol 1997; 169: 1655-1661

4 Jones SC et al. Magnetic resonance diffusion-weighted imaging: sensitivity and apparent diffusion constant in stroke. Acta Neurochir Suppl 1994; 60: 207-210

5 Koh DM, Collins DJ. Diffusion-weighted MRI in the body: applications and challenges in oncology. Am J Roentgenol 2007; 188: $1622-1635$

6 Takahara T et al. Diffusion weighted whole body imaging with background body signal suppression (DWIBS): technical improvement using free breathing, STIR and high resolution 3D display. Radiat Med 2004; 22: 275-282

7 Kwee TC et al. Diffusion-weighted whole-body imaging with background body signal suppression (DWIBS): features and potential applications in oncology. Eur Radiol 2008; 18: 1937-1952

8 Curvo-Semedo L et al. Diffusion-weighted MRI in rectal cancer: apparent diffusion coefficient as a potential noninvasive marker of tumor aggressiveness. J Magn Reson Imaging 2012; 35: 1365-1371

9 Padhani AR et al. Diffusion-weighted magnetic resonance imaging as a cancer biomarker: consensus and recommendations. Neoplasia 2009; 11: $102-125$

10 Sankowski AJ et al. The clinical value of MRI using single-shot echoplanar DWI to identify liver involvement in patients with advanced gas- troenteropancreatic-neuroendocrine tumors (GEP-NETs), compared to FSE T2 and FFE T1 weighted image after i.v. Gd-EOB-DTPA contrast enhancement. Med Sci Monit 2012; 18: MT33 - MT40

11 Hamstra DA, Rehemtulla A, Ross BD. Diffusion magnetic resonance imaging: a biomarker for treatment response in oncology. J Clin Oncol 2007; 25: $4104-4109$

12 Laun FB et al. Introduction to the basic principles and techniques of diffusion-weighted imaging. Radiologe 2011; 51: 170-179

13 Padhani AR, Koh DM, Collins DJ. Whole-body diffusion-weighted MR imaging in cancer: current status and research directions. Radiology $2011 ; 261: 700-718$

14 Le Bihan $D$ et al. MR imaging of intravoxel incoherent motions: application to diffusion and perfusion in neurologic disorders. Radiology 1986; 161: $401-407$

15 Lenz $C$ et al. Assessing extracranial tumors using diffusion-weighted whole-body MRI. Z Med Phys 2011; 21: 79-90

16 Ballon $D$ et al. Imaging therapeutic response in human bone marrow using rapid whole-body MRI. Magn Reson Med 2004; 52: 1234-1238

17 Hwang S, Panicek DM. Magnetic resonance imaging of bone marrow in oncology, Part 2. Skeletal Radiol 2007; 36: 1017-1027

18 Goodsitt MM et al. The composition of bone marrow for a dual-energy quantitative computed tomography technique. A cadaver and computer simulation study. Invest Radiol 1994; 29: 695-704

19 Hausmann $D$ et al. Whole-body MRI in preoperative diagnostics of breast cancer - a comparison with [corrected] staging methods according to the S 3 guidelines. Fortschr Röntgenstr 2011; 183: 11301137

20 Messiou C et al. Optimising diffusion weighted MRI for imaging metastatic and myeloma bone disease and assessing reproducibility. Eur Radiol 2011; 21: 1713-1718

21 Ley S et al. Neuroblastoma imaging. Fortschr Röntgenstr 2011; 183: $217-225$

22 Manenti G et al. Malignant renal neoplasms: correlation between ADC values and cellularity in diffusion weighted magnetic resonance imaging at 3 T. Radiol Med 2008; 113: 199-213

23 Woodfield CA et al. Diffusion-weighted MRI of peripheral zone prostate cancer: comparison of tumor apparent diffusion coefficient with Gleason score and percentage of tumor on core biopsy. Am J Roentgenol 2010; 194: W316-W322

24 Kwee TC et al. ADC measurements of lymph nodes: inter- and intra-observer reproducibility study and an overview of the literature. Eur J Radiol 2010; 75: 215-220

25 Thoeny HC et al. Combined ultrasmall superparamagnetic particles of iron oxide-enhanced and diffusion-weighted magnetic resonance imaging reliably detect pelvic lymph node metastases in normal-sized nodes of bladder and prostate cancer patients. Eur Urol 2009; 55: $761-769$

26 Lin G et al. Detection of lymph node metastasis in cervical and uterine cancers by diffusion-weighted magnetic resonance imaging at 3T. J Magn Reson Imaging 2008; 28: 128-135

27 Bonekamp S, Corona-Villalobos CP, Kamel IR. Oncologic applications of diffusion-weighted MRI in the body. J Magn Reson Imaging 2012; 35 : $257-279$

28 Ono $\mathrm{K}$ et al. Comparison of diffusion-weighted MRI and 2-[fluorine18]-fluoro-2-deoxy-D-glucose positron emission tomography (FDGPET) for detecting primary colorectal cancer and regional lymph node metastases. J Magn Reson Imaging 2009; 29: 336-340 
29 Chen $Y$ et al. The clinical application of whole-body diffusion-weighted imaging in the early assessment of chemotherapeutic effects in lymphoma: the initial experience. Magn Reson Imaging 2012; 30: 165170

30 Yagci $A B$ et al. The value of diffusion-weighted MRI for prostate cancer detection and localization. Diagn Interv Radiol 2011; 17: 130-134

31 Thormer $G$ et al. Diagnostic value of ADC in patients with prostate cancer: influence of the choice of b values. Eur Radiol 2012; 22: 1820

32 Hilario $A$ et al. The added value of apparent diffusion coefficient to cerebral blood volume in the preoperative grading of diffuse gliomas. Am J Neuroradiol 2012; 33: $701-707$

33 Lambregts DM et al. Tumour ADC measurements in rectal cancer: effect of ROI methods on ADC values and interobserver variability. Eur Radiol $2011 ; 21: 2567-2574$

34 Nakanishi $M$ et al. Relationship between diffusion-weighted magnetic resonance imaging and histological tumor grading of hepatocellular carcinoma. Ann Surg Oncol 2012; 19: 1302 - 1309

35 Moffat BA et al. Functional diffusion map: a noninvasive MRI biomarker for early stratification of clinical brain tumor response. Proc Natl Acad Sci U S A 2005; 102: 5524-5529

36 Patterson DM, Padhani AR, Collins DJ. Technology insight: water diffusion MRI - a potential new biomarker of response to cancer therapy. Nat Clin Pract Oncol 2008; 5: 220 - 233

37 Paran $Y$ et al. Water diffusion in the different microenvironments of breast cancer. NMR Biomed 2004; 17: 170 - 180

38 Sun YS et al. Locally advanced rectal carcinoma treated with preoperative chemotherapy and radiation therapy: preliminary analysis of diffusion-weighted MR imaging for early detection of tumor histopathologic downstaging. Radiology 2010; 254: 170 - 178

39 Dzik-Jurasz A et al. Diffusion MRI for prediction of response of rectal cancer to chemoradiation. Lancet 2002; 360: 307-308

40 Byun WM et al. Diffusion-weighted MR imaging of metastatic disease of the spine: assessment of response to therapy. Am J Neuroradiol 2002; 23: 906-912

41 Ballon $D$ et al. Bone marrow segmentation in leukemia using diffusion and T (2) weighted echo planar magnetic resonance imaging. NMR Biomed 2000; 13: 321-328

42 Reischauer $C$ et al. Bone metastases from prostate cancer: assessing treatment response by using diffusion-weighted imaging and functional diffusion maps - initial observations. Radiology 2010; 257: $523-531$

43 Messiou C, de Souza NM. Diffusion Weighted Magnetic Resonance Imaging of metastatic bone disease: A biomarker for treatment response monitoring. Cancer Biomark 2010; 6: 21 - 32
44 Hayashida $Y$ et al. Monitoring therapeutic responses of primary bone tumors by diffusion-weighted image: Initial results. Eur Radiol 2006; 16: $2637-2643$

45 Cui Y et al. Apparent diffusion coefficient: potential imaging biomarker for prediction and early detection of response to chemotherapy in hepatic metastases. Radiology 2008; 248: 894-900

46 De Keyzer F et al. Dynamic contrast-enhanced and diffusion-weighted MRI for early detection of tumoral changes in single-dose and fractionated radiotherapy: evaluation in a rat rhabdomyosarcoma model. Eur Radiol 2009; 19: 2663-2671

47 Larocque MP et al. Monitoring T2 and ADC at 9.4 T following fractionated external beam radiation therapy in a mouse model. Phys Med Biol 2010; 55: 1381 - 1393

48 Jung SH et al. Predicting response to neoadjuvant chemoradiation therapy in locally advanced rectal cancer: diffusion-weighted 3 Tesla MR imaging. J Magn Reson Imaging 2012; 35: 110-116

49 Kwee TC et al. Whole-body diffusion-weighted magnetic resonance imaging. Eur J Radiol 2009; 70: 409-417

50 Kawamura E et al. Clinical role of FDG-PET for HCC: relationship of glucose metabolic indicator to Japan Integrated Staging (JIS) score. Hepatogastroenterology 2008; 55: $582-586$

51 Picchio $M$ et al. Positive [11C]choline and negative [18F]FDG with positron emission tomography in recurrence of prostate cancer. Am J Roentgenol 2002; 179: $482-484$

52 Gulyas B, Nyary I, Borbely K. FDG, MET or CHO? The quest for the optimal PET tracer for glioma imaging continues. Nat Clin Pract Neurol 2008; 4: 470-471

53 Kwee TC et al. Complementary roles of whole-body diffusion-weighted MRI and 18F-FDG PET: the state of the art and potential applications. J Nucl Med 2010; 51: 1549-1558

54 Mori T et al. Diffusion-weighted magnetic resonance imaging for diagnosing malignant pulmonary nodules/masses: comparison with positron emission tomography. J Thorac Oncol 2008; 3: 358 - 364

$55 \mathrm{Ho} \mathrm{KC} \mathrm{et} \mathrm{al.} \mathrm{Correlation} \mathrm{of} \mathrm{apparent} \mathrm{diffusion} \mathrm{coefficients} \mathrm{measured} \mathrm{by}$ 3T diffusion-weighted MRI and SUV from FDG PET/CT in primary cervical cancer. Eur J Nucl Med Mol Imaging 2009; 36: 200 - 208

56 Schwenzer NF et al. Application of MR/PET in oncologic imaging Fortschr Röntgenstr 2012; 184: 780-787

57 Padhani AR, Miles KA. Multiparametric imaging of tumor response to therapy. Radiology 2010; 256: 348 - 364

58 Smith-Bindman R. Is computed tomography safe? N Engl J Med 2010; 363: $1-4$

59 Schaefer JF, Kramer U. Whole-body MRI in children and juveniles. Fortschr Röntgenstr 2011; 183: 24-36

60 Klasen J, Antoch G, Blondin D. MR imaging of the abdomen in pregnancy. Fortschr Röntgenstr 2011; 183: 514-522 